\title{
Análisis sobre los procesos de conflicto y tensión presentes en la transición social de género de niños, niñas y adolescentes (NNA) trans, desde la perspectiva de diferentes familiares vinculados/as a estos/as.
}

Analysis of conflict and tension processes present in the social gender transition of trans children and adolescents (NNA) from the perspective of different family members regarding these children and adolescents.

Análise sobre os processos de conflito e tensão presentes na transição social de gênero de crianças e adolescentes (NNA) trans, sob a perspectiva de diferentes familiares vinculados(as) a estes(as).

Sofía Alvarado*

\section{RESUMEN}

Las infancias trans han ido visualizándose en los últimos años, Palabras clave: debido a las voces alzadas de sus familias organizadas. Este artítransición social de culo se enfoca en los procesos de conflicto y tensión presentes en género, infancia, estereotipos de la transición social de niños/as o adolescentes trans, abordando géneros, familia. cadores y psicólogos/as). Parte del escrito pertenece a una tesis realizada en 2018, su análisis de contenido cualitativo muestra resultados orientados a la socialización familiar de la problemática, con discursos revestidos de tensiones entre los/as diversos/ as integrantes. Se concluye que existe inicialmente un rechazo a la identidad de género del/la NNA, en razón de estereotipos, imaginarios y adulto-centrismos. Finalmente, se interioriza la emergencia del infante de ser escuchado/a y reconocido/a, sin

* Chilena, Socióloga y Licenciada en Sociología de la Universidad de Playa Ancha, Valparaíso. sofia.alvarado.m@gmail.com 
derribar por completo las estructuras del género binario, provenientes de una estructura sexual de géneros reguladora de todas las relaciones sociales.

\section{SUMMARY}

Trans children have been visualized in recent years due to the raised voices of their organized families. This article focuses on the processes of conflict and tension present in the social transition of trans children or adolescents, addressing actors' experiences linked to them (family members, educators, and psychologists). Part of the writing belongs to a thesis carried out in 2018. Its qualitative content analysis shows results-oriented to the family socialization of the problem, with discourses coated with tensions between the various members. It is concluded that there is initially a rejection of the child's gender identity due to stereotypes, imaginaries, and adult-centeredness. Finally, the child's need to be heard and recognized is internalized without completely overthrowing the binary gender structures from a sexual gender structure that regulates all social relations.

\section{RESUMO}

As infâncias trans vêm sendo visualizadas nos últimos anos, devido às vozes erguidas de suas famílias organizadas. Este artigo enfoca nos processos de conflito e tensão presentes na transição social de crianças ou adolescentes trans, abordando as vivências de atores vinculados(as) a eles(as) (familiares, educadores e psicólogos(as)). Parte do texto pertence a uma tese realizada em 2018, cuja análise de conteúdo qualitativo mostra resultados orientados à socialização familiar da problemática, com discursos revestidos de tensões entre os(as) diversos(as) integrantes. Conclui-se que existe inicialmente um rechaço à identidade de gênero do(a) NNA, em razão de estereótipos, imaginários e adulto-centrismos. Finalmente, interioriza-se a emergência do infante de ser escutado(a) e reconhecido(a), sem derrubar por completo as estruturas do gênero binário, provenientes de uma estrutura sexual de gêneros reguladora de todas as relações sociais.
Key words: gender violence, students, agencies, intervention.

Palavras-chave: transição social de gênero, infância, estereótipos de gêneros, família. 


\section{Introducción}

El presente artículo sirve como testimonio sobre la realidad trans en la infancia bajo vivencias e interpretaciones, en las cuales diversos/ as agentes interactúan y se relacionan a estos/as en espacios íntimos, ya sea escuela, familia, sesiones, etc. Ello a partir de una visión cercana que le otorgan, en conocimiento y apoyo al proceso social de transición de género cuando se produce en la niñez o adolescencia. Los primeros ejes a investigar son los hitos importantes en la transición, los conflictos y tensiones sobre la situación de ser niño/a o adolescente trans en la sociedad, y los DDHH que le atañen a este grupo diverso.

Este escrito forma parte de una investigación mayúscula de pregrado titulada "Análisis sobre el proceso de transición social de género en niños, niñas y adolescentes trans, desde la perspectiva de diferentes actores (familia, profesionales de salud y educación) vinculados/as a estos/as". De esta manera, aquí se trabaja sobre tres de los cuatro ejes temáticos, pertenecientes al segundo objetivo específico de dicha tesis, que hace alusión a analizar procesos de tensión y conflictos encontrados en la transición social de NNA trans. La problemática principal tiene su base en cómo estaría operativa una norma y estructura sexual en el grupo de entrevistados/as, tanto en sus relaciones sociales internas como externas, donde se activan y aparecen una serie de dificultades en el reconocimiento y validación de "lo trans".

Para llevar a cabo la investigación, las aproximaciones teóricas y epistémicas utilizadas abarcan temáticas tomadas de estudios de género y teorías feministas sobre la familia, los roles de mujeres y hombres, y de cómo estos se interrelacionan al socializar las tareas que les competen a cada sexo/género. Del mismo modo, los resultados indagan en cómo el fenómeno trans interfiere en las diversas esferas de lo familiar, desde la teoría queer/feminista y la sociología del género, sumado a un acercamiento desde los aportes de la sociología y estudios de infancia, en que se identifica una subestimación hacia esta población falsamente homogénea y alejada de toda respuesta/ acción deliberativa. El artículo culmina con el análisis y resultados de las entrevistas realizadas a familias y profesionales, incluyendo los hallazgos levantados de este. 


\section{Contexto y problematización}

Las casi dos décadas de dictadura en Chile (1973-1990) marcaron un precedente en la historia de las mujeres y disidencias sexuales/políticas, por sus características como grupos violentados y con derechos nulamente alcanzados. En esos años, el Estado conservador, con una junta militar al mando, suprimió todo tipo de leyes en materia de reconocimiento social, debido a un interés de carácter económico que quedó evidenciado en la Constitución dictada por Augusto Pinochet en 1980. Como resultado, en los setenta y ochenta se generaron graves violaciones a los Derechos Humanos, con persecuciones políticas hacia personas que eran percibidas como amenaza u oposición al Gobierno Militar (Rojas, 2021). Estas no fueron el único foco de represión, dado que también las "minorías sexuales" sufrieron de manifestaciones de odio, asesinatos y arrestos. El aumento de las discriminaciones, sumado a la desinformación social de la época ante el tema - especialmente para con la comunidad trans-, dio paso a una clandestinidad con la que se desenvolverían las personas LGTBI+, afectando su dignidad de vivir, desarrollarse, trabajar, estudiar, etc., sin ser atendidas ni respetadas sus identidades de género ni orientaciones sexuales (OTD, 2012).

De esta manera, este grupo marginado por la sociedad chilena en dictadura tendrá conflictos judiciales, políticos y sociales, empeorados por el aprovechamiento de otras políticas e instituciones conservadoras, para ser arrestados/as o acusados/as de ir en contra de la moral. Como primera vía de reconocimiento, los organismos internacionales intervendrán a partir de la falta de resguardo en numerosos casos de violaciones a la integridad de personas LGBTI de nuestro país. El retorno a la democracia, en la década de los 90, tampoco logrará responder al problema mencionado, puesto que solo se abrirá hacia una experimentación piloto y paulatina de otras políticas sociales. Se justificará lo anterior, debido a la mantención de la Constitución Chilena de 1980 - hasta nuestros días-, con una transición política que tardará en reconocer, comprender e integrar a la diversidad sexual y sus derechos dentro de sus prioridades nacionales (Ibíd.).

En el año 2000 emergieron los primeros acuerdos y tratados internacionales para Chile, exigiendo su responsabilidad y cuidado en la inclusión, dentro del marco de respeto por la diversidad y la promoción 
de la no violencia, constituyendo definitivamente un avance respecto de lo producido en los años precedentes. Contrario a esto, en el mismo periodo y debido a sus postulados de "democracia en la medida de lo posible", la temática trans no fue incluida en la agenda nacional. Así, se siguieron produciendo detenciones policiales, violentando y reprimiendo a travestis, transgéneros y transexuales por el uso de sus vestimentas y actitudes no aceptadas en espacios públicos (Ibíd.).

Dos hechos cambian la historia nacional en lo judicial y legal. Por una parte, el caso "Atala Riffo y niñas vs. Chile", de la jueza Karen Atala y su familia en 2004, quienes fueron discriminadas por el Estado chileno. La situación, que posteriormente se conoció por la demanda a Chile ante la CIDH internacional, interpuesta por Karen Atala, culminó en un fallo victorioso en 2009. Además de conseguir la tuición de sus hijas, se declaró que, al ser este un país que no respeta los Derechos de Igualdad y no Discriminación, debía adherirse y aplicar las leyes internacionales, para velar y educar en pos del reguardo de personas LGBTI del territorio (Maturana, 2013).

Por otro lado está el caso de Daniel Zamudio y su asesinato en 2012: un crimen de odio y discriminación fundado en su orientación sexual. Este hecho desató la petición y dictación urgente de una ley "antidiscriminación" (Maturana, 2013). En su formalidad, la ley 20.609 vendría a sancionar a corto plazo, bajo procedimiento legislativo, todo acto de discriminación arbitraria. No solo castiga el acto violento producido por motivos de orientación sexual, sino por todo tipo de discriminación hacia toda identidad humana merecedora de respeto y derechos. Sin duda, se trata de un avance desde las percepciones sociales y sus relaciones, de un Chile que por primera vez se incorporó a la agenda internacional de DDHH y diversidad sexual (Mineduc, 2017).

En materia internacional, en 2006 un grupo de especialistas y diplomáticos de DDHH reunidos en la ciudad de Yogyakarta redactaron los Principios de Yogyakarta, que orientan y guían a los Estados, promoviendo derechos específicos de personas LGBTI. La "Aplicación de la Legislación Internacional de Derechos Humanos en Relación con la Orientación Sexual y la Identidad de Género" actúa a nivel mundial, evitando la acción deliberativa y separada de cada país. Con ello, se permite un funcionamiento en conjunto a los sistemas de la ONU y las diversas instituciones nacionales, esperando reducir los crímenes 
hacia la diversidad. Chile, como parte de este acuerdo, deberá guiarse por dichos principios cada vez que sea necesario ante un caso nuevo de violencia y vulneración de derechos. (Mineduc, 2017).

Ahora bien, entre los últimos acontecimientos está la promulgación de la "Ley de identidad de género", a finales de 2018, que permite apresurar los procesos de cambio de nombre legal y género en el registro civil para adultos/as trans. Antes de ser admitida, la ley pasó por largos procesos de validación y edición, en que importantes figuras y personas comprometieron y pidieron con urgencia su ratificación, para asegurar el resguardo de sus derechos. Para la abogada Ximena Guaché (2016), si bien anteriormente ya contábamos con una ley antidiscriminación, la misma no abordaba en específico la problemática del reconocimiento de la identidad trans, haciendo necesaria la aplicación de otra ley que se abriera a la realidad de un grupo social marcado por la discriminación y transfobia. Pero, pese a estar vigentes las leyes 20.609 y 21120 , la última concede estrictamente a adultos/as trans cambiar su nombre y sexo registral. De este modo, la niñez trans en Chile queda en un vacío legal que dependerá de la consideración de cada juez, aumentando las posibilidades de discriminación y vulneración de derechos de este grupo de infancias (Gauché y Lovera, 2019).

Como hito aún actual, considerando las situaciones adversas hacia NNA trans en espacios educativos, la Superintendencia de Educación (Mineduc, 2017) redacta las "Orientaciones para personas LGBTI" y la “Circular de educación para niños niñas y estudiantes trans $N^{\circ} 0768$ ”, acogiendo una serie de demandas trabajadas con diversas organizaciones de familias con NNA trans. Los documentos referidos muestran los tratados a los cuales Chile está adscrito, sugiriendo a todos los establecimientos incluir la temática trans dentro de sus lineamientos, como preparación previa ante algún caso con las mismas características. Allí se recomienda enfrentar las responsabilidades a nivel familiar y escolar, rigiéndose por el resguardo de la integridad del/la estudiante, el reconocimiento de su nombre social y su dignidad como persona de derechos (GLSEN \& Todo Mejora, 2019). La creación de los instrumentos señalados deja un registro en Chile en materia de infancias trans, al estar aprobados y promocionados como principios legales. En resumen, se materializa la visibilización y labor organizativa de las mismas 
personas que lo viven, mientras sigue en deuda la discusión concreta del proyecto de ley que integre a los/as menores.

Sobre el contexto de los últimos años en casos de NNA trans, la "Encuesta T" de OTD (2017) reúne 315 testimonios en los que se abordan diferentes temáticas asociadas a etapas en que cada persona se identificó como "trans. Como respuestas se tiene que el descubrimiento de la identidad y la puesta en marcha de la transición tardó, dependiendo tanto del proceso de aceptación como de cada vivencia. Para los/as entrevistados/as, los primeros indicios partieron desde un cuestionamiento personal y rechazo a las actividades relacionadas con su género desde la infancia temprana ( 0 a 11 años), aumentando en un conflicto en la adolescencia (de 12 a 18 años), cuando la problemática no fue reconocida a tiempo, sin poder ajustarse a lo impuesto o intensificándose, por consecuencia, la sensación de no pertenencia al género de nacimiento. Con ello, se expone la necesidad de analizar la infancia como momento clave en que aparecieron los primeros vestigios de rechazo y cuestionamiento de la identidad de género en los/as entrevistados/as.

$\mathrm{Al}$ ver cómo la transexualidad es identificada personalmente en la infancia, desde lo expuesto se entiende que se requiere una doble misión y cuidados en el presente: primero, al ser retratado como grupo de niños/as y adolescentes cuyos derechos humanos son protegidos $\mathrm{y}$, segundo, por formar parte de la comunidad LGBTIQ+, acogidos en Tratados Internacionales y Nacionales. Bien se ha hablado por separado de las minorías de edad, quienes están protegidas por los Derechos Internacionales y la Convención de Derechos del Niño, que rigen desde 1989, donde los Estados deben asegurar el libre goce y desarrollo de la niñez (Rodríguez, 2007). Por otro lado, sobre la realidad de la "diversidad sexual", especialmente la adulta, estos se acogen por las leyes antidiscriminación, Identidad de Género, Principios de Yogyakarta y la ONU. Sin embargo, pese a ser avances, ambos factores no han logrado cruzarse para la creación de una ley de identidad de género nacional que sea inclusiva con las infancias y adolescencias trans, lo que deja un vacío en el acto de auxiliar ante discriminaciones hacia dicha población (Mineduc, 2017).

Se presenta aquí la incongruencia y problemática trabajada por Gauché (2016) en materia de derechos y defensa de los derechos humanos de la comunidad de NNA LGBITQ+, pues puede volverse noci- 
va al producirse en espacios íntimos de estos/as, donde se relacionan periódicamente. Así, las violencias y diversas situaciones de vulneración sufridas por este grupo se agravan, impidiendo el libre desarrollo del menor y rompiendo con tratados internacionales alcanzados. Sumado a ello, se puede reflexionar que, debido a la tardanza de políticas y la falta de prioridad de la problemática, se dificulta aún más la subsistencia de las infancias, al contar con menores herramientas que un/a adultos/a para su resguardo.

Es allí donde el entorno influirá primordialmente en el desarrollo de estos/as niños/as pues, al estar excluidos/as, carecerían de visibilización e interés público, con datos verdaderamente desalentadores. No es precisamente lo trans lo que lleva a estar mal a un/a niño/a, sino los actos de vulneración y no reconocimiento constantes, los que quebrantan su formación como persona. Contrariamente, cuando se permite a un niño/a transitar social y legalmente en sus espacios, desenvolverse libremente como desea y a su ritmo, haciendo presente sus necesidades, escuchando su voz, se logra un proceso de crecimiento sin cargas negativas ni rechazos sociales (Gauché y Lovera, 2019). Asimismo, el apoyo familiar se daría desde la provisión de un espacio seguro y la permisión de una libre exploración de identidad o manifestaciones de género, evitando el cuestionamiento de algunos/as familiares, especialmente abuelos/as, antes o después de una transición (OTD, 2017). En otros casos, el mismo entorno, al no estar consciente/preparado para la "nueva identidad", al negarla frente al uso indebido de palabras y tratos, podría generar violencias y discriminaciones, que son necesarias de abordar y hacerse cargo (Rubio, 2009).

Respecto del entorno cercano en que viven los/as sujetos/as trans, Vacas (2014) evidencia en su investigación el desconocimiento de la transexualidad, a través de la seguridad y negación con la que sus entrevistados/as afirmaron no tener a una persona transexual dentro de sus integrantes familiares. Así, a diferencia de las otras categorías LBTIQ_, lo "trans" representa menores porcentajes de "conocimiento y visibilidad", ya sean mujeres u hombres transexuales. Según la autora, son los mismos/as transexuales quienes se autoexcluyen y prefieren no ser visibles, siguiendo una lógica de camuflaje para evitar las discriminaciones. 
En un estudio de OTD (2017) se entregan resultados que sostienen cómo las personas entrevistadas se identifican como trans desde edades tempranas, y no solamente con una identidad de género distinta a la de sus compañeros/as semejantes, sino que también sus gustos o preferencias hacia ejercicios muchas veces tenían que ver con "actividades consideradas del otro género". A su vez, Dinamarca (2014) analiza el reconocimiento de estereotipos y del orden del género desde la infancia, relacionados al "deber ser" en función de los sexos. Con ello asocia un conjunto de "creencias, reglas, expectativas y atribuciones" de lo que es ser mujer y hombre socialmente. Esto recae y se reproduce mediante la crianza, especialmente en el grupo más joven de una familia.

Y es que existe algo mayor, que causaría exclusiones justificadas desde el género cultural y lo biológico. Para Robles (2012), aquello biológico, demostrado desde lo que la ciencia nos dice sobre el sexo y su composición química, estaría asociado tanto a lo femenino como a lo masculino mediante consideraciones culturales transmitidas por la socialización de los géneros. Estos géneros buscan y se validan a través de prácticas y pruebas, tanto materiales como subjetivas, teniendo lugar en la producción y normalización de manifestaciones sexuales. A su vez, Butler (2006) expone que entre los géneros hegemónicos y el poder sexual no existirían otras variaciones que no sean hombre o mujer, polaridades extremas que aparecen para clasificarnos y obedecer al sistema regulador.

De esta manera, se presentan breves nociones del contexto nacional de quienes han vivido una vida rechazando la norma social sobre el supuesto de hombre/mujer, sin poder concordar con las reproducciones y mantención de las prácticas que se le asocian involuntariamente. Para los grupos trans, al tener una identidad de género opuesta a lo establecido, se generan marginaciones que dificultan el reconocimiento social/político, y aún más cuando situamos esta realidad en menores de edad. Con el paso del tiempo, dicha comunidad sigue resistiendo entre vacíos legales, junto a medidas que han emanado del trabajo colaborativo, y que resuelven parcialmente los problemas a los que son expuestos/as - violaciones a su dignidad e integridad-, venidos especialmente desde el entorno cercano y constante. A partir de ello nacen nuestros primeros cuestionamien- 
tos en torno a cómo se desarrolla el apoyo a nivel familiar y escolar, cuál es el rol de la familia en dichas infancias, qué conflictos y problemáticas resultan al permitir el desarrollo y transición de género en este tipo de niñez, y qué derechos sirven como soporte para la subsistencia de estas de familia.

\section{Perspectiva teórica y estado del arte}

\section{Género, roles y familia.}

\section{Género/sexo y roles en la sociedad}

En el mundo social que vivimos, género y sexo se interrelacionan directamente bajo estereotipos, actitudes y roles que se han otorgado para categorizar, enumerar, ordenar, trabajar, asociar, etc., a individuos/as. Poco conocidos son los espacios neutros o intermedios entre hombres y mujeres, en los que las personas puedan optar o expresar discrepancia frente a este tablero con reglas e instructivos ya escritos. Todo cuerpo, por tanto, estará subyugado y determinado por el género cultural que le pertenece o le asignan, obedeciendo y estableciendo un desarrollo que responda a este propósito.

Para adentrarnos en la comprensión de "eso" que va por sobre el género, en la teoría de Judith Butler (2006) se defiende la existencia de un poder regulador, que somete a las personas subjetivadas por un sistema sexual-político. Una norma cultural dentro de este sistema intentará destacar las diferencias sexuales entre dos únicos sexos - femenino/masculino - que conviven y se muestran en calidad de cuerpos estables, relacionados diferenciadamente, asociados al deseo heterosexual, sin espacio a otras manifestaciones.

Las características que permean al género y sus atributos estarían mayormente asociadas a aquello social, más que hacia lo biológico, pues, si bien esto último es importante, lo cultural primaría hacia todo lo que vendría después, acompañado de estereotipos, imaginarios, concepciones. etc. A lo referido, se coincide con que: "Hablar de género es referirse a un tamiz cultural, a una identidad y a un conjunto de prácticas, creencias, representaciones y prescripciones sociales (Ávila, 2019 p.5; Lamas, 2016) Podríamos decir que el factor social del género hace que no podamos considerarlo como personal, sino generado por 
colectividades, al atribuir aquellas significaciones mediante prácticas que variarán según el contexto.

Pese a que el género se vive como la identidad personal, está allí desde antes de nosotros/as y permanecerá con su entramado cultural, social y de poder. La razón se debe a que interiorizamos, aprendemos y funcionamos pensando que es nuestro, sin percatarnos que este no funciona ni se activa, sino desde la percepción del otro. Para Butler (2006), lo que se piensa como una capacidad propia para crear la realidad individual, estaría constituido por configuraciones externas. Sumado a esto, el género también constituye un propio régimen de regulación y disciplina en sí mismo, como operación implícita dentro de la sociedad y sus relaciones; este va normalizando y definiendo las prácticas a seguir para integrarse al sistema. De modo que la norma definiría lo "inteligible" y "no-inteligible", pese a que algunos/as individuos/ as desplieguen sus máximos esfuerzos para ausentarse de ella, todo acaba por pertenecer sin deliberación al sistema material del cuerpo.

Lo anterior lo aborda Soley-Beltrán (2009), argumentando que habrá corporalidades menos aceptadas y rechazadas por hacer visible su diferencia de género, al no concordar frente al dualismo hombre/ mujer con las leyes de género-sexo. En el otro extremo, "lo abyecto" direccionado hacia lo no-inteligible dentro de un espacio inhabitable persiste en el sistema normativo social desde la marginación social. En este sentido, pese a que la categoría nombrada se encuentra desde un nivel jerárquicamente bajo y sin posibilidades de cambiar, la producción y existencia de personas en esta posición no será menor. Así, el antagonismo entre lo "normal" y "lo abyecto", emergidos del mismo sistema, será necesario para la existencia y funcionamiento de la sociedad.

El sistema sexo-político de los géneros se justifica generando y reproduciendo categorías binarias estrictas relacionadas con un tipo de sociedad y normas, mediante un discurso de peso cultural social que castiga, rechaza, categoriza la materialización de los cuerpos en las relaciones sociales. Ante ello, como opción des-configuradora, se presenta el acto de re-pensar las categorías transmitidas, consideradas bajo una realidad sujeta. Estas serían más bien moldeables en un proceso de cambio constante y de búsqueda de reconocimiento como individuos/as sociales todos/as (Butler, 2006). Así mismo será para den- 
tro de lo abyecto: algunos/as aprovecharán este espacio para resistir y ver las posibilidades políticas como performáticas de burlarlo desde adentro (Soley-Beltrán, 2009).

Para Robles (2012), el género funcionaría en tanto dimensión del sexo, proporcionando imaginarios al igual que otros tipos de reglamentos derivados del poder y manifestados en discursos encargados de inducir a los/as sujetos/as a una serie de normativas reguladoras, motivadas por un fin político de demostrar asimetría. Las diferencias partirán por subordinar históricamente a la mujer con el hombre, asimismo, de la viabilidad de unos cuerpos por sobre otros. Esta postura se asemeja a la concepción de Butler (2006) desde el sistema sexual y las nociones de cuerpos inteligibles y no inteligibles. Hablamos de un aparato social que incluye y excluye a los/as individuos involuntariamente, a partir de un conjunto a manifestaciones biológicas, psíquicas y sociales.

\section{La familia y el género}

Desde la sociología de la familia y el estudio de ésta, el objeto de estudio del presente artículo se sitúa ligándose al género y los elementos transmitidos a partir de padres, familia y niños/as. La crítica se basa en cuestionar la reproducción de roles diferenciados dentro de este núcleo, la perspectiva del "niño/a" en la sociedad y el familismo de los Estados modernos. Asimismo, se busca indagar la construcción y educación de NNA como la lucha por hacer posible la agencia y reconocimiento de los derechos humanos, como personas independientes y heterogéneas.

Refiriéndonos a la familia, se comprende que, dentro de sus relaciones, esta no abarcaría únicamente el mero acto de adiestramiento y cuidado de niños/as, sino también una gran base educativa y de transmisión de valores, como de socialización entre padres, madres e hijos/ as. Dio Bleichmar (2009) desarrolla un importante aporte sobre cómo se dan las relaciones familiares, principalmente en el fenómeno de la "hija y la madre", en el que se percibe a esta última como "sujeta" diferenciada socialmente desde su género con los hombres. Ello mientras sucede algo parecido en las relaciones "hijo/ padre", alejadas en su interacción y significaciones del grupo femenino. Tanto niña como niño pasan por procesos de asimilación e incorporación de representacio- 
nes transmitidas por la madre o padre - expectativas, deseos, modos de relaciones, entre otros-, que permiten el desarrollo constante de su identidad nuclear y su género.

Se suma a ello que la estructura intersubjetiva configuradora de las identidades estaría abierta, psicológica y físicamente, a cambios generacionales, pues contiene características de búsqueda personales y mutaciones constantes que transitan desde que el niño/a nace hasta la llegada de su adultez (Dio Bleichmar, 2009). No solamente la apariencia forma parte de la manifestación aprendida del género, así también las actitudes y expresiones corporales visibles de lo masculino/femenino. Por otro lado, se encuentra también el proceso de identificación que hace el niño/a en el aspecto subjetivo. Así, el lenguaje, la comunicación y los códigos sociales se interpretarán personalmente acercándose más a un género y alejándose del otro (Dio Bleichmar, 2010).

Hijos/as se identifican y comparan con sus padres/familiares cercanos, desde representaciones conscientes e inconscientes, hacia una personificación asociada a la posición de interacción con los demás. De tal modo, se incorpora un tipo de socialización e intersubjetividades a las que se relaciona su modelo de género paternal o maternal a seguir. El proceso de reconocimiento e identificación, no solo de género sino también otras categorías, es donde la sociología ha querido indagar bajo sus distintas epistemologías, precisamente por construirse desde el momento en que nos relacionamos con otros/as (Ibíd.).

Lourdes Gaitán (2021) cita a Prendergast (2000) respecto de la concepción del cuerpo y el género en el cambio de infancia a adolescencia. Este transcurso personal y algo colectivo, percibido en familia y colegio, ocurre física y psicológicamente como una condición social conflictiva, al ser natural pero sorpresiva. El paso a un cuerpo menos estático/ambiguo - de niño/a - se moldeará a la manera de una identidad femenina o masculina - de adulto/a - binaria y notoria. Por ende, las mujeres advierten el paso a ser mujeres por su menarquia, y los niños al ser conscientes de sus cambios debido a un acercamiento profundo con sus pares. Ambos géneros sufrirán transiciones y percepciones de cambios, acompañados de la vergüenza, el secreto, los pudores y sensibilidades. Asimismo, en lo personal existirá una prolongación o visualización temporal de la identidad personal, luego de ver las manifestaciones de género en otros/as semejantes o no. 
Desde un discurso experiencial, Paul B. Preciado (2019) evoca cómo padres y madres buscan proteger las normas sociales por sobre los intereses del "niño", transmitidas generacionalmente a través de la intimidación y el castigo de la desviación, limitando la autodeterminación y desarrollo de este. En este sentido, se busca mantener viva la ley moral, exacerbando las diferencias corporales y gestos femeninos/masculinos, donde la infancia es un dispositivo de validación del "adulto" y lo normativo es la heterosexualidad. Dicha yuxtaposición tendría una base naturalista, religiosa y política que ve en peligro el sistema y, por ende, ejerce la pedagogía de la muerte, reprimiendo bajo lemas de "defensas y derechos" de la familia y la infancia.

A lo largo de los apartados se articulan miradas más abiertas al descubrimiento de los cambios y la adaptación de manifestaciones de género, consolidándose de mano con la comparación de grupos símiles/contrastantes - en que pareciera tratarse de procesos conscientes-, encontramos la parte más oscura de un sistema disciplinado y causante de estos/as futuros/as adultos/as. Consecuentemente, en casos en los que hay identidades o expresiones de género que no coinciden con lo normativo, el núcleo familiar puede ser represivo con el NNA o, inversamente, podrá ser comprensivo al identificar y permitir el desarrollo de otras identidades, teniendo en su contra un sistema fuerte de normas y comprobaciones para su subsistencia.

\section{Nuevas familias y roles}

La modernidad implica la aparición de nuevos prototipos familiares, en los que las infancias y mujeres han pasado a cumplir una mayor función participativa, a diferencia de siglos y décadas pasadas. Para Elizabeth Jelin (2010), el matrimonio, conocido por su asociación con objetivos de negociación y adquisición de bienes, actualmente ha modificado sus bases ya no solo para crear una unión institucional entre dos personas, sino también para ligar otros factores y nuevas inclusiones de personas como son los/as hijos/as. Así, se han visibilizado nuevos tipos de familias diversas, compuestas por una figura maternal e hijos/as, o por un padre e hijos/as, o por abuelos/as y nietos/as, y cuantas variaciones existan, relacionadas o no por parentesco. A estas nuevas tipologías, que rompen con las antiguas formas de agrupación histórica, que la autora define como "familia contemporánea", se suman las mutaciones de sus miembros/as, como ha sido el caso de la 
incorporación de la mujer a la actividad laboral, el reconocimiento de voz y el derecho de NNA, la definición de la edad de inicio laboral, la jubilación en personas mayores, entre otros factores.

Empero, pese a la apertura social ya comentada, persisten roles que dificultan el equilibrio armonioso de las relaciones generacionales y sobre todo de género: las niñas siguen relacionándose con actividades de casa y de cuidado de sus hermanos/as, para ayudar a sus madres cuando estas necesitan salir a trabajar. Mientras las mujeres adultas persisten en dar provisión, en ocuparse de niños/as y ancianos/as en casa, sumado a la responsabilidad del trabajo doméstico y la doble jornada (Jelin, 2020). Todos estos casos fraccionan las estructuras familiares y la democracia colaborativa interna de un hogar, al no permitir un equilibrio de quehaceres para todas las partes (Jelin, 2010).

En compensación, ha habido una mutación de la figura mantenedora de lo económico de una familia - o jefe de hogar-, al ser ahora las mujeres adultas quienes también trabajan y mantienen sus hogares, aunque diariamente sigan realizando las labores domésticas en la esfera privada, sin dejar la categoría de ama de casa y cuidadora. Sabemos que una familia no se sostiene ni alimenta exclusivamente del aporte económico, también las tareas de casa y de "subsistencia" son vitales, por lo que todavía las responsabilidades de este espacio no logran ser recíprocas por parte de los varones (Jelin, 2020). Así, existe más de un tipo de reproducción generada por el grupo femenino: la biológica — gestación de hijos/as, fecundación y aumento demográfico-, la reproducción de la fuerza de trabajo -labores de mantenimiento y aseguración de subsistencia de los trabajadores para darles fuerza-, y finalmente la reproducción social mantenimiento del sistema social— que trata la transmisión de valores y conductas esperadas, el cuidado de la familia y la socialización temprana de niños/as. (Jelin, 2020; Larguía y Dumoulin, 1976).

La crítica va en que las nuevas posibilidades económicas y laborales de las mujeres se han sobrecargado, sin una reestructuración de los roles ni de participación doméstica, por ende, las relaciones familiares persisten en desequilibrio. Todo cambio dependerá de las negociaciones que se puedan llevar a cabo en cada familia, entendiendo que la base de una democracia familiar incluye labores y derechos que responsabilizan a todos sus miembros ya sean niños/as que estudien, padres/adultos/as que trabajen y la inclusión de adultos mayores. 
La infancia, desde la figura del " $h i j o / a$ ", se presenta como un individuo en construcción, con una determinada tarea - reducida hasta su independencia - "cooperativa" en razón de su género y edad, como sucede también con los/as ancianos/as. Mientras niños y niñas son influenciados por los cambios de la familia contemporánea, de la educación y del trabajo que sus miembros cumplen, se amplía la concepción de niñez. Por contraparte, se sigue suponiendo que ciertas labores destinadas a los/as hijos/as serán a modo de inversión de su futuro y su adultez, dependiendo de si son hombres o mujeres (Jelin, 2010).

Para Rodríguez (2007), el discurso del familismo, en el que lo paternal es lo privativo y a la vez autoritario, enfundado por la labor de las instituciones y el Estado, resulta una mayor división entre el mundo adulto/ niño. Con base en la historia de la infancia y la familia ha existido una diferencia notable entre los Estados antiguos y modernos: los primeros, con una visión un tanto vaga del concepto "infancia", sin tomar en cuenta las responsabilidades morales de las "familias" y acelerando la mayoría de edad del "menor" bajo obligaciones. Por el contrario, los modernos han enfocado una mirada más evolucionada hacia la labor de controlar, vigilar y disciplinar a estos/as para cuando se conviertan en adultos/as, limitando por tanto, las acciones mientras persiste condición de "niño/a". Las visiones positivas de la modernidad han conllevado a un cambio de pensamiento, con base en la protección y socialización que el NNA debe ir adquiriendo. Como dualidad, aparece un adulto-centrismo que contempla a esta población en construcción — de racionalidad y estabilidad-como seres débiles, en un estado temporal inestable pero igual para todos/as.

Finalmente, podemos agregar que, tal como Oakley presenta el mito de la familia, todavía subsisten las ideas de familia del siglo XX, debido a una relación de falsa felicidad, democracia, apoyos, confidencialidad y lealtad entre sus miembros. De tal manera, la realidad de la unión familiar contempla "desigualdades, inequidades y abusos encubiertos" que recaen negativamente y en mayor cantidad hacia las mujeres y niños/as, en dependencia y opresión mutua. Esto diferencia a los hombres, que se mantienen en una condición más favorecida, tanto por el sistema patriarcal como por la estructura vertical a la cual representa lo económico y social (Gaitán 2021; Oakley, A. 1993). 


\section{El fenómeno trans en la sociedad}

\section{Lo trans}

Puede que exista una confusión entendible cuando se habla de "trans", "transexualidad", "transgénero", "travesti”, y es debido a que se han encasillado todas las definiciones dentro de una gran “T”, que deja espacio a la duda y a la desinformación. Así, será necesario tratar y diferenciar aquí al menos los tres primeros conceptos antes de comprender la infancia trans y el fenómeno social y familiar que se vive en ella.

Para comenzar, la transexualidad se liga ontológicamente a procesos biomédicos, quirúrgicos y psicológicos, con una correspondencia directa hacia la modificación física de la persona, para la adquisición de su nuevo rol de género, vivido internamente y con anterioridad. Así se detalla en la siguiente definición: “Transexual como aquella persona que interfiere en su cuerpo a nivel hormonal y/o quirúrgico, con el fin de convertirse en un sexo diferente" (Soley-Beltrán, 2009, p. 265). Sobre lo expuesto, para una persona transexual, un "sexo diferente" alude al opuesto binario, encajado en los estándares y conformidades de las normas sociales. Lo corporal, requiriendo hacer caso a la identidad interna, pasará por numerosas intervenciones, consideradas como "reasignaciones de género", para la asimilación de una imagen femenina o masculina. De tal manera, el cuerpo, como materia moldeable y receptiva, buscará adecuar mente y género interno, donde reside lo subjetivo, representado en la identidad esencial de una persona (Soley-Beltrán, 2009).

En cambio, "ser" una persona transgénero, ha sido equivocadamente reconocido como periodo previo de la transexualidad y la intervención médica, pero este concepto más bien abordaría una situación estable en sí misma. Lo transgénero trae consigo una "identidad de género" y búsqueda del "yo" que no coincide con el sexo biológico de nacimiento (Mineduc, 2017). Este término, a su vez, se relaciona directamente con la persona no- cisgénera, con una expresión de género contraria a la impuesta socialmente, a una transición libre y diferida de la orientación sexual:

... las personas transgénero, que son aquellas personas cuya identidad de género difiere de su sexo biológico. Estas personas pueden ser mujeres transgénero, que desde lo masculinx transitan a lo fe- 
meninx u hombres transgénero, que desde lo femeninx transitan a lo masculinx. Las personas transgénero pueden ser heterosexuales, homosexuales o bisexuales. Normalmente, su identidad y/o expresión de género no se corresponde con las normas y expectativas sociales, y en el caso de las personas transgénero, no se altera o no desean cambiar los caracteres sexuales con los que nacieron. (Todo Mejora, 2017, p. 40)

La cita anterior asume en lo transgénero una definición amplía de posibilidades sexuales, sin la necesidad de intervenir en lo hormonal o físico, como lo sería en una persona transexual. El paso de quien encarna esta identidad sucedería desde una transición al género sentido, direccionada hacia lo social y la apariencia.

En otros casos, transgéneros/as serían aquellos/as que han decidido habitar sin estándares sexuales impuestos, cuestionando los ideales de cuerpos y rechazando simultáneamente las concepciones o roles relacionados al género entre lo masculino y femenino. Esto último, pertenecería a un grupo más bien disidente, bajo una condición subversiva del sistema (Vendrell, 2009) Por lo tanto, esta investigación se refiere a lo "trans" tomando el segundo concepto de transgénero, por ser más generalizado (Todo Mejora, 2017), y referido a todas las personas y procesos cuya identidad de género y expresión de género no corresponden con las normas y expectativas sociales.

Ahora bien, volviendo al proceso de transición —social, físico, corporal o legal一, este será totalmente personal y diferente en relación a plazos y sometimientos, tanto para individuos/a trans como para transexuales. Así también debemos entender que no todas estas identidades trans optarán por una transición. Para Butler (2006), la transición es parte de un cambio llevado por la persona para ser percibida legal, médica o socialmente desde el género al cual realmente pertenece e identifica, "para vivir como tal". Igualmente, la transición se valida mediante la comprobación de otros/a al permitir tratar a las personas por el género que expone el cuerpo o documento que lo avale.

Existen otras visiones, como la de Vacas (2014), que expresan una perspectiva más crítica sobre las intervenciones y la transexualidad, ya 
que estos recursos serían la opción más simple y menos innovadora, al buscar la posibilidad de cambio de otro género que también estaría instaurado en el sistema. Por otro lado, sentirse trans - y no transexual- correspondería a una actitud más combativa, de lucha social y transgresión, que ampliaría nuevas posibilidades de géneros sin una transición definida. En este sentido, se polarizan las visiones y sus procedimientos, las concepciones de los géneros y las maneras de situarse ante la vida social.

A su vez, pese a su visión más contestataria sobre el sistema, Vendrell (2009) argumenta que querer modificar el cuerpo pasa por una elección personal de tipo "asimilacionista", existiendo colectivos que acogen y ven la medicina como remedio a sus incertidumbres. Puesto que la decisión estribaría en algo propio, como búsqueda legítima de armonía, ciertos/as individuos/as necesitarán realizar una transición, con el fin de entrar en espacios antes rechazados, comprendiendo Vendrell la necesidad de querer integrarse a la sociedad y ser aceptados/ as; como también alude Butler (2006) en párrafos anteriores, sobre el reconocimiento social de los otros en sociedad.

\section{Infancia trans: derechos}

\section{Infancias trans}

De aquí en adelante nos referimos a la importancia de la familia, adultos/ as cercanos/as e infancias que manifiestan ser trans, dentro de relaciones que se desplazan cotidianamente entre el colegio y el hogar. Ambos espacios, frente al conocimiento de la situación, actuarán aceptando la condición del NNA — buscando ayuda de especialistas e información-, o, contrariamente, negando y haciendo caso omiso de lo expresado. En este apartado se cruzan la condición de niño/a, los derechos humanos y el reconocimiento de la identidad de género de este grupo.

Dinamarca (2014) enfoca su estudio sobre la trayectoria trans, reflexionando acerca del rol de la familia, la crianza y cuidado de infancias. Allí los discursos de personas trans, ya adultas, testimonian una identidad de género percibida a temprana edad, momento desde el cual aparecieron las disconformidades del género asignado ante el cuerpo biológico. Se evidencia que, para ese entonces, las herramientas que tenían sus familias para abarcar el tema no fueron las más idóneas, a pesar de haber manifestado desde niños/as su real género 
sentido, por lo que no hubo soluciones reparadoras. La adolescencia se caracterizó por agravar las contradicciones y manifestaciones de descontento de los/as jóvenes, quienes, al ver su desarrollo físico en una dirección no esperada, interiorizaron sus cuestionamientos sin referentes que les guiaran personalmente. Por otro lado, sus familias, al no entender la situación, ocuparon gran tiempo en la búsqueda de diagnósticos oportunos, tardando en encontrar respuestas.

Para APA (2011), la opción de vinculación de padres con sus pares puede servir como una de las acciones que velan por el desarrollo de las infancias y sus resguardos, en unión con instituciones que procuren tratar la temática trans del grupo etario. Esto mientras se acepta que un/a menor con "género atípico" vive una condición alejada de ser una "fase" transitoria. Vacas (2014) también afirma cuán importante es asociarse con personas afines a la causa trans, como aporte para conseguir una mayor presión social. Lo anterior conlleva a aceptar y conocer otras manifestaciones de género existentes, cuestionar las distinciones de roles y estereotipos, que muchas veces se realizan y discriminan al/a la menor pasándole a llevar en su propio espacio.

Sin duda, el núcleo familiar constituye un factor decisivo en la contención del/de la menor al presentarse un conflicto de este tipo; asimismo, para encontrar el apoyo y primeras respuestas, evitando las consecuencias psicológicas y emocionales que traen estas infancias incomprendidas por la negligencia adulta, el sistema sexual de género y la falta de información del medio.

\section{Labor de padres/ madres frente a una infancia trans y la protección de sus derechos}

Si bien siempre han existido identidades trans, estas han emergido con mayor notoriedad en la última década dentro la esfera pública, frente a casos de familias que lo viven con sus NNA. Son precisamente éstas quienes han debido buscar apoyo entre otras familias con mismas realidades, investigando bibliografía internacional para perseguir y construir un futuro más alentador en lo que respecta a derechos, leyes y proyección de vida.

Los padres de niño/as trans tienen incidencia, de cuidado, responsabilidad y tarea de representación del NNA, principalmente por permitir el desarrollo de sus deseos y su autonomía progresiva, como por 
ejemplo en la elaboración del protocolo y de la ley de identidad para infancias. Así, familias y adultos/as deben dar la discusión, exponiendo y resguardando en pos del reconocimiento de estas infancias, sobre todo a partir del campo jurídico y social que aportará mayores garantías. Paralelo a esto, siempre se debe contar con la opinión directa de NNA en cuestión, en cuanto sean conscientes de sus "derechos" frente al Estado (Gauché y Lovera, 2019).

El reconocimiento legal de estas infancias debe asegurar y concretar un futuro digno, avanzando ya no únicamente desde un discurso revestido por miradas médicas —ni diagnósticos ni patologización-, sino en vías de crear uno que cuestione la ausencia de sus derechos humanos, de la integridad, de la libre identidad de NNA, de su deseo superior y capacidad de agencia, de temáticas que han sido silenciadas por años al alejarse de la norma y lo hegemónico. Comenzamos a hablar sobre la emergencia con la que se debe tratar esta realidad, cuando tiene que ver con la dignidad de un grupo social considerado "minoritario" y a su vez "homogéneo", no solo por ser trans, sino debido a ser menores de edad e infantes. Gracias a casos de infancias diversas, se ha evidenciado un cambio paradigmático y epistémico en el que puede haber tantos tipos de NNA como variedad de familias existentes (Guerrero y Muñoz, 2018).

Se puede identificar cómo se entrelazan los roles de adultez e infancia desde una posición hegemónicamente asimétrica y jerarquizada, provenientes de un mundo social que, pese a comenzar a fijar su mirada en la niñez, tendría una perspectiva adulto-centrista. Gauché y Lovera (2019) critican por un lado, cómo el Estado les protege por ser menores de edad y, por otro, les suprime, patologiza y oculta el derecho autónomo a expresar y vivir su género, o bien su orientación sexual.

En relación al Estado, no se puede omitir la labor de mantención de la norma y la hegemonía político-sexual, sumada a la represión de las libertades, llevada a cabo hoy en día por grupos extremistas y religiosos, oponentes a nuevas legislaciones pro-derechos LGBTIQ+. Estos/as "defensores/as" han ideado y abanderado la lucha por los derechos de una infancia, bajo un prototipo rígido que supone en este grupo la heterosexualidad y la géneronormatividad. Con ello, "el niño" al que apelan tendría un cuerpo -órganos, fluidos, genitales- que le corresponde exclusivamente a la familia, pero privándole 
a éste del derecho de poder decidir y gobernarlo individualmente, a través de una educación de la norma sexual y de género. Dicho esto, la idealización de la infancia se materializa en la modelación de los cuerpos y sus manifestaciones, a la reducción de las subjetividades, a la amenaza a la resistencia y la campaña del terror a las desviaciones. El niño imaginario, con una familia reducida a madre y padre, sirve de argumento para la protección de la "vida", legitimando el rechazo a otras identidades como el "niño queer". Bajo estos principios, la interrogante es: ¿qué sucede con las infancias diferentes? ¿Quién las defiende y da el derecho a libre elección de su género y sexualidad sin violencias y discriminaciones? (Preciado, 2019).

Siguiendo con esto, se expone cómo NNA LGBT tendrían mayor probabilidad de presentar enfermedades mentales y, con ello una tendencia alta al suicidio - cuatro veces más que otros niños/as - visto por ellos/as como solución debido a la falta de herramientas. La causa principal sería el rechazo que muchas veces reciben en establecimientos no seguros ante incidentes de violencia, o dentro de familias que omiten las discriminaciones, afectando su bienestar personal. Así, la estigmatización dificultaría el desarrollo y las estrategias sociales de estos/as niños/ as, hacia conductas destructivas y depresivas. Entre otras consecuencias, por falta de atención a la salud mental de parte de autoridades y familias, están los bajos niveles de autoestima, sumado a un ausentismo escolar (Todo Mejora, 2016).

Para llevar la problemática a una proyección, Rubio (2009) exhibe las circunstancias en las cuales un/a transexual vive, percibe y siente discriminaciones, las que son innumerables a lo largo de su vida. Estas situaciones en que se les excluye, se deben a una cultura social legitimada que impone la heteronormatividad tanto a hombres como mujeres, en la que toda identidad sexual diferente sería propensa a marginación, exclusión y rechazo de parte de la sociedad. La barrera primaria de rechazo sería la familia y los vínculos cercanos; la secundaria pertenecería al trabajo y la escuela - con sus creencias sociales y religiosas-, generan una sucesión de discriminaciones y exclusiones "retroalimentadas" circularmente. Esto genera que las personas trans - $\mathrm{y}$ en especial NNA-, sean propensas a desarrollar dificultades en cuanto a inclusión laboral, social y académica, y generalmente optan por encerrar su identidad y realidad trans. 
Respecta de la sociología de la infancia, la emergencia ante el desarrollo de un campo que trate específicamente dicha población expresa también una falta de atención social al objeto de estudio y a su capacidad de agencia (Rodríguez, 2007). Pareciera que no existe información suficiente, de investigación científico-social y actividades relacionadas, que aporten a la academia un espacio sociológico sobre la infancia. Existen avances en estudios y nuevas aproximaciones, formuladas desde escuelas internacionales, inglesas o europeas; pese a ello, al estar escritos en otros idiomas, aumenta el desconocimiento de los avances y las dificultades en la comprensión de las diversas infancias entre países de habla hispana y de Latinoamérica o Chile (Gaitán, 2021).

\section{Metodología}

La presente investigación es de enfoque cualitativo (Alonso, 1998), con un paradigma interpretativo (Valles, 2007), de tipo fenomenológico y diseño flexible (Vasilachis, 2006).

Descripción del contexto y muestra

Familias y Profesionales de Educación y Salud vinculados/as a casos de infancia trans, residentes de Santiago, Viña del Mar, Quilpué y Valparaíso.

\section{Cantidad de entrevistas}

12 personas, entre ellas cuatro profesionales (dos psicólogas + dos educadores) y ocho familiares directos (tres padres, dos madres, dos abuelas, una tía).

Técnica de producción de datos

Entrevista en Profundidad (Valles, 2007)/semiestructurada (Alonso, 1998)

Técnica de análisis de datos

Análisis de contenido cualitativo (Cáceres, 2003).

Calidad del dato

Credibilidad/confiabilidad o dependibilidad/empoderamiento (Vasilachis, 2006).

Aspecto Ético

Consentimiento informado. Participación voluntaria. 


\section{Plan de análisis}

El plan de análisis de la información se realizó tomando como modelo a Cáceres (2003), basado en Mayring (2000), y los seis pasos para el análisis de contenido cualitativo. Se parte seleccionando el objeto de análisis dentro de un modelo de comunicación, seguido por el desarrollo de un preanálisis, la definición de las unidades de análisis, el establecimiento de reglas de análisis y códigos de clasificación, el desarrollo de categorías, terminando por la integración final de los hallazgos.

\section{Análisis y resultados}

Los resultados de esta investigación logran dar respuesta al interés fundamental de la realidad que viven familias y adultos/as cercanos/as a NNA trans, concretamente sobre nuestro segundo objetivo específico elegido de esta investigación: "Analizar los procesos de conflicto y tensión en torno a la transición de género de NNA trans desde la perspectiva de diferentes actores vinculados/as a éstos/as". En el análisis, cada eje temático (3) contiene una categoría. Se debe precisar que, para este artículo, se decidió mostrar únicamente aquellos ejes y categorías de la matriz que reflexionaran acerca de los conflictos y tensiones, dando cuenta de las problemáticas más complejas que se reflejan en la tabla de análisis adjunta:

OBJETIVO ESPECIFICO No2: Analizar los procesos de conflicto y tensión en torno a la transició de género de NNA trans desde la perspectiva de diferentes actores/actrices vinculados/as a éstos/as

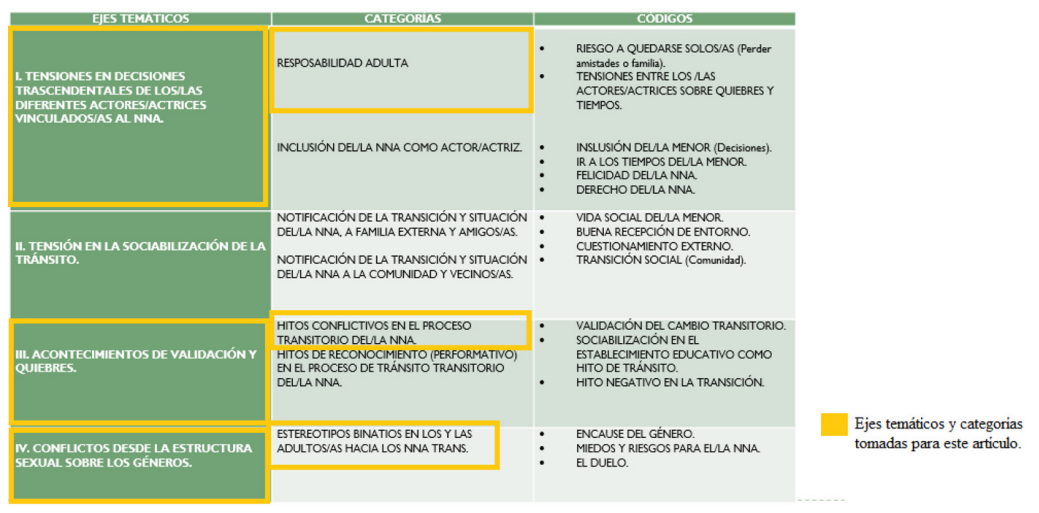




\section{Primer eje temático: tensiones en las decisiones trascendentales} de los diferentes actores vinculados/as al NNA

El primer eje temático, procuró atender las tensiones dentro de las decisiones más importantes entre los/as entrevistados/as, analizando los testimonios referidos a cómo se planificaron las diferentes estrategias de aceptación, inclusión y tránsito de la "nueva identidad" del NNA en los espacios en que este/a se relacionaba. El presente título, acerca de las tensiones en las decisiones trascendentales, se desplazó englobando la problemática de "responsabilidad de los/as adultos/as", categoría primordial para comprender cómo sucedieron posteriormente las transiciones y cómo lo percibió el entorno directo de NNA. Sin embargo, aun cuando hablamos del primer proceso, este se presenta como uno de los más complejos y cargados de "fricciones" para los/as integrantes, debido a su rol de representación y cuidado de quien expresa la nueva identidad de género.

\section{Categoría: responsabilidad de los/as adultos/as}

En virtud de las entrevistas realizadas, sucedió que, para el proceso de decisiones y estrategias para la reinclusión del NNA en sociedad, fue necesario que todos los/as actores vinculados/as a este/a - adultos/ as o no- buscaran las instancias necesarias antes de proceder a un cambio. Asimismo, se debió llegar a un consenso relacionado a cómo permitirían esta apertura y qué papel les tocaba vivir como familia y entorno cercano, pensando en el bienestar del/de la menor. Este momento se reconoce por iniciar con un cuestionamiento sobre los roles de género, generacionales y estereotipos sociales, pasando directamente a las permisiones y derechos existentes para las infancias, en todos los espacios de desenvolvimiento social.

Dentro de los relatos recogidos, padres y familiares hablaron de la transición a partir de las decisiones que debieron realizar, considerando en orden las más prioritarias para ayudar a su niño/a o adolescente. En ello, primero se expresaron las preocupaciones en torno a la responsabilidad como adultos/as de estabilizar lo repercutido a nivel familiar, al momento de enterarse que su hijo/a o familiar era trans. Como lo expone Rodríguez (2007), los cuidados y el paternalismo de la infancia demuestran un adulto-centrismo social que impera en primeras instancias de decisiones, viendo culturalmente al "infante" como 
un ser débil y por tanto desprotegido/a, donde padres y adultos/as familiares son quienes llevan la conducción y decisión en el grupo.

Seguido de esto, en las entrevistas se presenta un cambio de acción en profesionales y familias al dar apoyo y acompañamiento superior al NNA, sumado al escuchar, atender y brindarle bienestar hacia su deseo integral como persona, mencionado también por Gauché y Lovera (2019). La mayoría de las decisiones descritas partieron haciendo alusión al acompañamiento y tránsito desde los pasos que iba dando el/la menor, y de cómo éste/a manifestaba sus tiempos y elecciones. Así se explica que la impaciencia y apuro sobre los procesos subjetivos y físicos del tránsito, fueron caracterizados con mayor fuerza por el grupo de menores en cuestión, al ser estos quienes lo vivían personalmente. Tales situaciones fueron diferenciadoras entre adultos/as y NNA, pero superadas a través de la empatía y la comprensión conjunta desde la esfera familiar. De tal modo, desde estos primeros cambios positivos y sustanciales los/as menores se volvieron protagonistas y motor colectivo de validación, mientras sus familias salían adelante con su responsabilidad de protección y aceptación de la "nueva" identidad.

Un factor que les diferencia de otras tipologías de familias, fue vivenciar conflictos y reconocer la problemática desde una perspectiva experimental, avanzando bajo intentos paulatinos de hallar medidas en pos de su hijo/a o pariente. Se une a lo que Dinamarca (2014) evidencia en su estudio, pues sin duda es una realidad disímil de otras infancias e identidades hegemónicas. Así, al no contar con preparación previa para distinguir el fenómeno trans, y pese a la carencia de atención y ayuda profesional, los/as entrevistados/as manifestaron ocupar grandes periodos en buscar soluciones rápidas como medida inicial. Por último, las familias participantes destacaron una fuerte intención de superación de frente a los desafíos en dicho periodo, y que la mejor medida hallada fue la interacción, el transcurso del tiempo y la intención de consenso entre las partes.

Es menester testificar cómo todos/as nuestros/as informantes en algún momento dijeron haber visto al NNA deprimido/a o con problemas evidentes sobre su autoestima, teniendo que tomar en cuenta y remediar desde la acción inmediata, comenzando por lo que implicaría la aceptación e identificación de sus principales necesidades. Esto se relaciona con el informe de Fundación Todo Mejora (2016), en el que 
queda constancia que las condiciones nocivas a las que un/a menor de edad trans puede estar expuesto/a cuando su familia no lo/la apoyaomite, margina y discrimina-, tendrían efectos sobre su salud mental. Las conductas autodestructivas y el aumento de posibilidades de padecer depresión se presentaría en NNA LGBTQ+ con mayor fuerza, y nuestras entrevistas lo corroboraron.

En el caso de los/as profesionales entrevistados/as, éstos/as manifestaron que tuvieron de reconocer los derechos del/de la menor, destacando la educación y un buen entorno social, que debieron ser reestablecidos y ofrecidos en un espacio de acogida adecuado para la situación, así como lo refieren Guerrero y Muñoz (2018). Como actores importantes, reforzaron, desde su profesión de salud o educacional, el carácter "humano" que legitimaba al NNA como persona con una identidad de género que debe ser respetada, aun siendo menor de edad. De esta manera, uno de los profesionales (3) declaró que la primera opción, tanto para profesores/as, como psicólogos/as y familia, fue atender y acompañar, entregando todos los recursos para la inclusión y desarrollo del menor, transmitiéndole seguridad, cuidado y respeto por la diversidad. Una de las mayores responsabilidades nombradas por estos/as profesionales, fue autoeducarse y actualizarse acerca del tema, resguardando el cumplimiento de sus derechos o dando aviso cuando existía una vulneración o violación de la integridad del/de la menor.

Con lo anterior, surge desde los profesionales un replanteamiento de lo que significaría ser "diferente", ayudando al/a la estudiante en cuestión a comprenderse como un "otro" - nombrado en las entrevistas-, al enfrentar una existencia cuando no se cumple con las normas y, por ende, aislado/a, violentado/a y rechazado/a en razón de patrones sociales establecidos. En efecto, lo que propuso uno de los/ as entrevistados/as sería una suerte de "resistencia/ ruptura", como sucede con lo abyecto para Soley-Beltrán (2009), al volver el término "otro" hacia una herramienta en que nos asumimos en nuestra posición de repensar las identidades, como Butler (2006), o considerar que las manifestaciones cambian a través de las generaciones, como Dio Bleichmar (2009).

Finalmente, uno de los primeros quiebres entre el grupo de familiares y profesionales fue sobre el interés y visibilización de los 
procesos del/de la menor. Ante ello, Gauché (2016) enfatiza respecto de cómo se generarían las carencias y principales violencias dentro del ámbito público y privado de un NNA trans, que disminuyen con el apoyo hacia los cambios que este/a va solicitando. Al romper la primera barrera de discriminación, descrita por Rubio (2009) - la familia-, la acción de estos/as adultos/as fue respetar/acompañar, desde lo físico, lo psicológico y lo social. Un factor importante se basó en la necesidad de comunicar desde el núcleo hacia otros/as actores secundarios/as cuando todo estaba preparado y adecuado para ello. Así, se delineó un momento complejo y valioso que implicó adherirse a las demandas de sus niños/as dejando de lado las propias, situando la adultez desde un papel nuevamente complementario, al estar dispuestos/as a enfrentar dialogando toda duda externa relativa a la problemática en el camino.

\section{Segundo eje temático: acontecimientos de quiebres del proceso transitorio en actores vinculados/as al NNA trans}

Nos encontramos con el segundo eje temático, en el que se subraya las circunstancias enrevesadas por hechos e hitos conflictivos que pusieron en tensión a los diferentes entes responsables de los/as menores, tanto familiares como profesionales de salud/educación. A continuación se ve cómo se asumieron y compartieron roles, apoyando la "nueva identidad" y "haciendo frente", mientras persistían aún cuestionamientos internos y/o dudas de personas externas hacia el NNA en cuestión.

\section{Categoría: hitos conflictivos en el proceso transitorio del NNA}

Esta categoría apunta a los hitos negativamente mencionados por los/ as entrevistados/as, debido a dudar justamente cuando pensaban ya haber pasado las mayores dificultades e impedimentos.

Los/as informantes hablaron sobre los momentos asociados a tensiones y quiebres, en que, como familia, debieron nuevamente decidir por el bienestar del NNA conflictuado/a. Así, se demostró que pensar en los aprendizajes y experiencia adquirida hasta ese entonces les sirvió de herramienta para lidiar con los nuevos obstáculos. Este tema fue analizado como una categoría aparte, puesto que los/as entrevistados/ as presentaron recurrentemente hechos negativos que se disputaban 
con los positivos desde sus vivencias. Pasar por ello les permitió reconocer a quienes impidieron la transición del niño/a, al acentuar el género asignado, desestimando por ende la real identidad del/de la mismo/a. En el caso de las familias, estas relataron haber podido salir de las situaciones problemáticas gracias al alcance de información y de argumentos aprendidos sobre la base de derechos de infancia y género.

Encontramos que parte de estas problemáticas nacieron a partir de concepciones binarias y conservadoras de los géneros, con las cuales las familias, muchas veces, sin encontrar una solución, prefirieron como primeras medidas- alejarse de aquello que les vulneraba. Así, los principales conflictos emergieron en un comienzo, cuando intentaban normalizar/asimilar el género preferencial del NNA como opción más cercana para su bienestar. Pero la existencia de un poder regulador, representado en personas y normas culturales de género-sexo, les hacía más difícil su propósito (Butler, 2006). Estos hitos marcaron fuertemente la manera como establecieron las relaciones, más aún cuando, en la mayoría de las ocasiones embarazosas, el/la menor se encontraba presente. Como también lo expone Robles (2012), dentro de las relaciones entre "dos géneros" del poder, se presentan prácticas que serán invalidadas socialmente cuando no quepan dentro de la normalidad femenina y masculina, o cuando sobrepasan lo instaurado. De esta manera, los/as entrevistados/as expusieron las dificultades que aparecieron luego en el camino de la transición de sus niños/as, cuando intentaban mantener una armonía positiva/tolerante frente a otras opiniones cerradas al cambio, en respuesta de una norma social fija y polarizada que castiga advirtiendo lo ambiguo o discordante.

Por último, se analiza cómo la opción del tránsito para las familias entrevistadas, pese a ser considerada una de las más indicadas y aceptadas, contó con mayores obstáculos, dificultando las relaciones y la formación autómata sobre la temática trans en todo momento. Vacas (2014) explicita que la transición producida de un género a otro estaría calificada como la "más fácil", puesto que no se innovaría o cuestionaría el sistema. A su vez, cuando se trabajó desde la infancia, hubo ciertos contrastes, pues los casos presentados tuvieron que reorganizar y habituarse a un "nuevo género" del niño/a o adolescente de la manera más tranquila y pacíficamente posible, ya que no implicaban procesos 
de hormonización ni operaciones, como sí sucede en adultos/as trans, cuando esta es su elección. Pese a esto, en el contexto nacional la transición social en niños/as sigue siendo la única opción estática a la que se puede acceder, ya que se les privaría en materia legal de una inscripción a la ley de identidad de género. De modo que no se concibe la posibilidad de transición legal como parte de los derechos otorgados/ pertenecientes a las infancias (Gauché y Lovera, 2019).

Por consiguiente, aun cuando todos los NNA referidos/as en nuestros casos transitaron - respondiendo a un orden binario-, su trasformación para la sociedad sigue siendo un hecho excepcional y menos prioritario, a diferencia de adultos/as trans, o bien de otras realidades de niños/as cisgéneros/as. Esto causaría que la realidad y necesidad de expresar el género sea todavía desaprobada, incomprendida y no reconocida socialmente.

Tercer eje temático: conflictos desde la estructura sexual de los géneros

El último eje temático, se desarrolló mediante el análisis de los estereotipos de género que reproducían la estructura y norma binaria, presentes en los discursos de las familias y profesionales de esta investigación, en las diversas instancias. Sumado a ello, fue posible conocer cómo lo binario traspasaría los demás ejes temáticos, especialmente con el anterior, apareciendo nocivamente al momento de decidir y aceptar una decisión.

\section{Categoría: estereotipos binarios en los/as adultos/as hacia los NNA trans}

Para la siguiente categoría se analizaron relatos de conflictos en hitos significativos que generaron quiebres en las relaciones y en momentos previos/relativos al tránsito, debido a catalogarse como experiencias "negativas" o "trabas".

Un argumento generalizado por algunos/as actores fue el haber tenido una visión machista, conservadora, religiosa o biologicista, que recriminaba repudiando el actuar del NNA en sus primeras manifestaciones. Desde estas tensiones percibidas, profesionales y familias tuvieron que partir reconstruyendo las nociones aprendidas por una educación heterosexual y géneronormada, concientizando lo que Pre- 
ciado (2019) propone en torno a los géneros. Ello sumado al cambio del tipo de niñez, que se logró dando espacio a las subjetividades y la independencia del cuerpo del NNA, con una formación más libre y menos privativa de la familia.

Aun así, la mayoría de los NNA en discusión, al no presentar manifestaciones propias de su "sexo" y preferir actividades del género contrario desde su infancia primaria - OTD (2017) lo advierte como rasgo común-, fueron discriminados/as rápidamente por algunos/ as de sus familiares directos/as. Las entrevistas describen discusiones producidas culpando a otros/as adultos/as por influenciar e incentivar al menor en su actuar "anormal". Se suma a esto que el tránsito del NNA no fue posible hasta que dichos actores - reacios a la situación- lo aceptaron al profundizar sobre las prácticas personales y creencias de carácter social — como lo expresa Lamas (2016) en Avila (2019) —, que no daban espacio a tolerar variaciones sobre lo que se considera ser hombre o ser mujer.

Un ejemplo de ello se da cuando uno de los entrevistados (padre 3), habiendo reconocido y vivenciado el desarrollo de su hija, sufrió persistentemente embates personales por los estereotipos y las creencias heteronormadas/binarias. Como tal, el padre, al presenciar a su "hijo" vestido con un disfraz de "niña", describe implícitamente cómo se activaron en él mecanismos diferenciadores de lo femenino y masculino. Allí es donde la norma corregidora se impuso, creando en él disgusto y rechazo, pese a ser su hija quien lo hacía. Así, para Preciado (2019) la infancia trans, al no ser hegemónica y por tanto "queer", recibe la discriminación de la norma y sus dispositivos al desviarse disidentemente del supuesto de modelo de "niño" protegido. Igualmente, Vendrell (2009) justifica sobre una norma que no permite variaciones corporales ni sociales, promocionando dos extremos totalmente opuestos que tienen por objetivo estar separado el uno del otro. Esta situación, dada en otros casos, condujo a seguir rechazando y negando por largo tiempo la identidad de género del/de la menor de parte de padres, abuelos, tíos, primos. Atendiendo a lo anterior, se deduce cómo una disputa personal por algo culturalmente aprendido, termina ahondando en la relación que se tenía con los demás como con la menor.

A su vez, la denominación "el duelo", referida al proceso simbólico de dejar ir a un hijo/a por la llegada de otro/a, se encuentra mayor- 
mente asociado a los actores masculinos - padres y abuelos-, quienes relataron haber demorado mucho más en recuperarse y razonar, a diferencia de las mujeres. Así, tal como se presenta en el estudio de OTD (2017), aparecen familiares - en este caso abuelos- que retrasan la transición antes pero también durante el mismo proceso. Tías, abuelas y madres entrevistadas expresaron haber comprendido en su totalidad la situación, debido a una tenacidad en querer acompañar al/a la niño/a hacia la transición social y psicológica, dando los soportes, cuidados y defensas, aun cuando la problemática no estaba solucionada ni definida del todo. Se refuerza la "labor de cuidado", que Jelin (2010) y Jelin (2020) exponen sobre el grupo femenino y la prolongación del éxito personal de ellas por atender las necesidades de los demás.

Por otro lado, la implicación que tuvo el conflicto desde las parejas fue puesta en discusión, al producir rupturas/separaciones matrimoniales en varios casos, debido tanto a la falta de mediación en las opiniones - especialmente del grupo masculino-, como a la imposibilidad de armonizar y democratizar el problema y tareas entre toda la familia (Jelin, 2010). Como consecuencia, se rompe con el "mito" de la familia: de estabilidad, apoyo mutuo e igualdad, afectando negativamente a mujeres y niños/as del núcleo (Gaitán, 2021; Oakley, 1993).

\section{Conclusiones}

Respecto de todo lo mencionado en el análisis, se pudo considerar la importancia del último eje temático por sobre los demás en la vida de los/as entrevistados/as, como barreras desplegadas constantemente. Los enfrentamientos aparecieron para reestablecer las normas que buscaban validarse, primordialmente por conspirar en contra de la mantención de la familia y la de una sociedad géneronormada. Se concluye que dichas situaciones responden a una estructura de estereotipos de género, en la que los hombres tuvieron mayores problemas para deconstruir los valores aprendidos cultural y generacionalmente, y adecuarse a una nueva realidad para la cual no estaban preparados en lo más mínimo. Lo anterior no deja de reconocer los apremios convertidos en superación y máximos sacrificios desplegados especialmente en madres y abuelas. 
Se expone una complejidad en los entrevistados/as en poder superar sus propios constructos y estereotipos, al vivir una transición de género infantil sin apoyos institucionales, legales, psicológicos y emocionales que permitieran llevar la vivencia de mejor manera. Así, en primera instancia, las temáticas fueron abarcadas adulto-céntricamente, debido al desconocimiento de los Derechos del Niño y lo Trans, para luego informarse y conllevar empíricamente la lucha como familia, pudiendo llevar a una inclusión paulatina en las decisiones y acciones del/de la menor. De mano de lo anterior, se expone una permanencia de la condición del NNA derivando en incertidumbre, que mejoró mediante decisiones colectivas y una autoformación de todos los miembros.

Por último, se descubrió que los conflictos y tensiones, además de tener una base educativa hegemónica desde cuestionamientos externos, estaban asociados a visiones naturalistas y religiosas extremistas -católicas o evangélicas-, produciendo retrasos constantes en la reorganización familiar. Sin embargo, aun cuando las transiciones referidas fueron netamente sociales - de nombre social y vestuario, a diferencia de una transición adulta-, se reconoce en todo momento la fuerza de la norma sexo-género del sistema, que genera discriminaciones, falta de apoyo e invisibilidad legal social de dichas realidades y sus familias.

Otros hallazgos no incluidos en los ejes

- Familia/escuela serían los primeros entes notificadores de conductas alejadas de la norma sexual.

- Población entrevistada principalmente profesional, con niñas trans en su mayoría.

- Falta de estudios sobre la percepción infantil de la temática trans.

\section{Referencias}

Alonso, L. E. (1998). La mirada cualitativa en Sociología. España: Fundamentos.

Álvarez-Gayou, J. (2003). Cómo hacer investigación cualitativa. Fundamentos y metodología. México: Paidós Educador.

APA. (2011). Respuestas a tus preguntas sobre individuos transgéneros e identidad de género. Asociación de Psicología Americana. Segunda Edición, 1-6. 
Avila, A. (2019). Psicoterapia con perspectiva de género: Indagando posibilidades dentro del psicoanálisis contemporâneo. Scielo. Artigos. Sex., Salud Soc. (Rio J.) (33), 118-136.

Butler, J. (2006). Deshacer el género. Barcelona: Paidós Studio.

Cáceres, P. (2003). Análisis cualitativo de contenido: una alternativa metodológica alcanzable. Psicoperspectivas, (2), 53-58.

Dinamarca, C. (2014). De la transgresión a la normalización: Análisis de trayectoria social de personas transexuales desde la sociología de la desviación. Valparaíso: UPLA.

Dio Bleichmar, E. (2010). Diversidad Sexual. Cuestionario a Emilce Dio Bleichmar. Aperturas Psicoanalíticas, 38.

Dio Bleichmar E. (2009). Las teorías implícitas del psicoanalista sobre el género. Panel "Teorías implícitas de los analistas sobre la feminidad". Congreso IPA, Chicago, 2009. Aperturas Psicoanaliticas, 34 .

Gaitán, L. (2021). El enfoque de género en los estudios de infancia. Grupo de Sociología de la Infancia, 1-27.

Gauché, X. (2016). Proyecto: "Sistema de garantías de los derechos de la niñez", Boletín $\mathrm{N}^{\circ}$ 10.315-18, Santiago, Chile.

Gauché, X. y Lovera, D. (2019). Identidad de género de niños, niñas y adolescentes: Una cuestión de derechos. Revista Ius et Praxis, 25(2), 359-402.

Guerrero McManus, S., y Muñoz, L. (2018). Epistemologías transfeministas e identidad de género en la infancia: del esencialismo al sujeto del saber. Revista Interdisciplinaria de Estudios de Género de El Colegio de México, 4(1), E168.

GLSEN \& Todo Mejora. (2019). Una crisis global en el clima escolar: perspectivas sobre estudiantes lesbianas, gays, bisexuales, transgénero y queer en América Latina. Santiago, Chile: Todo Mejora.

Jelin, E. (2010). Pan y Afectos. Buenos Aires: Fondo de Cultura Económica de Argentina.

Jelin, E. (2020). Las tramas del tiempo. Familia, género, memorias, derechos y movimientos sociales. Antología Esencial. Buenos Aires: CLACSO.

Lamas, M. (2016). “Género”... En: MORENO, Hortensia \& ALCÁNTARA, Eva (coords). Conceptos Clave en los Estudios de Género Volumen 1. México: CIEG. p. 155-170 
Larguía, I. y Dumoulin, J. (1976). Hacia una ciencia de la liberación de la mujer. Barcelona: Anagrama.

Maturana, C. (2013). Chile: vulneración y desigualdad de derechos por orientación sexual. "Diversidad Sexual en Chile y el Mundo". Santiago, Chile: Le Monde Diplomatique.

Mineduc. (2017). Orientaciones para la inclusión de personas Lesbianas, Gays, Bisexuales, Trans e Intersex en el sistema educativo chileno. Santiago, Chile: Ministerio de Educación de Chile.

Oakley, A. (1993). Women and children first at last: Parallels and Differences between Children and Women Studies en Childhood as a Social Phenomenon: Lessons from an International Project. Eurosocial Report 47, 51-70. Viena: CentroEuropeo.

OTD. (2017). Encuesta T. Primera Encuesta para personas trans y género no conforme en Chile. Informe Ejecutivo, (I), 1-38. Santiago de Chile.

Preciado, P. (2019). Un departamento en Urano: Crónicas de cruce. Barcelona: Anagrama.

Prendergast. (2000) "To become dizzy in your turning": Girls, bodymaps and gender in childhood ends. The Body, Childhood and Society. London: Macmillan.

Robles, R. (2012). Construcciones de los cuerpos de la niñez internada en la década del dos mil. Una aproximación desde el género. Santiago, Chile. Universidad de Chile.

Rodríguez, I. (2007). Para una sociología de la infancia: aspectos teóricos y metodológicos. Madrid: CIS, Centro de Investigaciones Sociológicas.

Rubio, F. J. (2009). Aspectos sociológicos de la transexualidad. Madrid: Nómada.

Rojas, N. S. (2021). Movimientos de mujeres en Chile y el camino a una constitución feminista. Anuari Del Conflicte Social, (10), 21-60.

Soley-Beltrán, P. (2009) Transexualidad y la Matriz Heterosexual: un estudio crítico de Judith Butler, Barcelona: Bellaterra.

Todo Mejora. (2016). Encuesta Nacional de Clima escolar. Experiencias de violencia e inseguridad escolar estudiantes Lesbianas, Gays, Bisexuales y Trans. Santiago, Chile: Fundación Todo Mejora.

Todo Mejora. (2017). Enseñando diversidad. Manual de apoyo a profesores, tutores y apoderados para enseñar sobre diversidad, 
orientación sexual e identidad y expresión de género a niñxs y adolescentes entre 9 y 12 años. Santiago, Chile: Fundación Todo Mejora.

Vacas, A. I. (2014). Hacia la integración social: una propuesta comunicativa para él. Valladolid: Universidad de Valladolid. Facultad de Ciencias Sociales, Jurídicas y de la Comunicación.

Valles, M. (2007). Técnicas Cualitativas de la Investigación Social. España: Editorial Síntesis.

Vasilachis, I. (2006). Estrategias de Investigación Cualitativa. España: Gedisa.

Vendrell, J. (2009). ¿Corregir el cuerpo o cambiar el sistema? La transexualidad ante el orden de género. Sociológica (Méx.), 24(69), 61-78. 